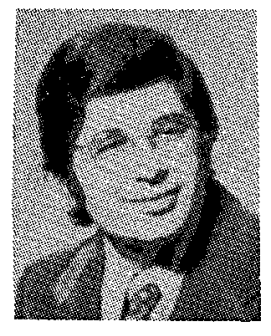

applications.

Dr. Pack is a member of Tau Beta Pi, Phi Kappa Phi, Omicron Delta Kappa, and the Operations Research Society of America.

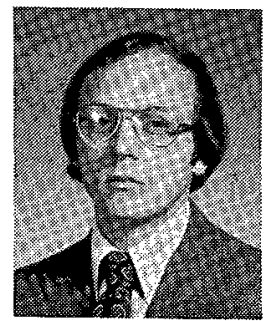

Bruce A. Whitaker was born in Caroleen, NC, on July 13, 1945. He received the B.S. degree in applied mathematics from North Carolina State University, Raleigh, in 1963, the M.S. degree in operations research from The Johns Hopkins University, Baltimore, MD, in 1968 , and $\mathrm{Ph} . \mathrm{D}$. degree in operations research from New York University, New York, in 1974.

$\mathrm{He}$ has worked on various aspects of traffic network planning since joining Bell Laboratories in 1967.

Dr. Whitaker is a member of the Operations Research Society of America, Pi Mu Epsilon, Phi Kappa Phi, and Phi Eta Sigma.

\title{
On the Transmitted Power in Generalized Partial Response
}

\author{
JAMES E. MAZO, MEMBER, IEEE, AND JACK SALZ
}

\begin{abstract}
The advantages with regard to noise enhancement of certain suboptimum nonlinear techniques for "equalization" of linear distortion on channels used for digital data transmission have recently received considerable attention. These nonlinear schemes fall into two classes: decision feedback and nonlinear precoding. Yet each of these methods has an associated peculiarity which has impeded attempts to give firm bounds on performance. Thus, for decision feedback error propagation has caused the analytical pains, while for nonlinear precoding of an $L$-level alphabet the unknown increase in transmitter power has been culpable. The former problem has recently received successful attention by one of the present authors and some colleagues. Here we address the concomitant problem for nonlinear precoding and its extension to quadrature amplitude modulation (QAM), and show that the transmitted normalized power $P$ satisfies $\left(L^{2}-1\right) / 3 \leqslant$ $P \leqslant\left(L^{2}-1\right) / 3+1$.
\end{abstract}

\section{INTRODUCTION}

$\mathbf{M}$ IYAKAWA AND HARASHIMA [1] and Tomlinson [2] have suggested a nonlinear data precoding scheme for eliminating intersymbol interference caused by past data symbols in pulse-amplitude modulation (PAM) digital communication systems. ${ }^{1}$ Partial response techniques [3], [4] constitute a special case. Price [5] optimized such a system operating over a noisy frequency attenuating channel, while implementation simplifications have been considered by Messerschmitt [6].

The purpose of this paper is to examine this precoding scheme and its extension to quadrature amplitude modulation (QAM). We pay particular attention to the transmitted signal power when independent symbols are encoded in this nonlinear manner. This question has heretofore been neglected

Paper approved by the Associate Editor for Data Communication Systems of the IEEE Communications Society for publication without oral presentation. Manuscript received July 11, 1975; revised October 2,1975 .

J. E. Mazo is with Bell Laboratories, Murray Hill, NJ 07974.

J. Salz is with Bell Laboratories, Holmdel, NJ 07733.

${ }^{1}$ We have sometimes heard these schemes being called the "Tomlinson filter" and sometimes the "modulo-transmitter (MT)" technique. and our results provide tight bounds on the average transmitted signal power.

We begin with a brief review of the precoding scheme which is best explained with the aid of a block diagram shown in Fig. 1. The output symbols $\left\{y_{k}\right\}$ which are the input to the channel obey the nonlinear recursion

$$
\begin{aligned}
y_{k} & =f\left[x_{k}-\sum_{i=1}^{N} \beta_{i} y_{k-1}\right], & k & =1,2, \cdots \\
& =0, & k & =0,-1,-2, \cdots
\end{aligned}
$$

where the function $f(u)$ is defined as $f(u)=u$ if $|u| \leqslant L$; otherwise $u$ is to be reduced to this range by algebraically adding an appropriate multiple of $2 L$ and then the result being called $f(u)$. Also in (1), $\beta_{i}$ are real constants and $x_{k}$ is the data symbol modeled as an $L$-valued random variable ( $L$ fixed throughout) which takes successive values independently and with equal probability from the set

$$
\begin{aligned}
\pm 1, \pm 3, \cdots \pm(L-1) & \text { if } L \text { even } \\
0, \pm 2, \cdots \pm(L-1) & \text { if } L \text { odd. }
\end{aligned}
$$

The utility of this filter in data transmission can be explained by considering the filter output sequence $\left\{y_{k}\right\}_{1}{ }^{\infty}$ to represent data symbols which are transmitted by PAM over a linear time-invariant channel. Let the overall impulse response, including transmitting and receiving filters, be $h(t)$. The system output sample at time $k$ is then given by

$$
\begin{aligned}
s_{k} & =\sum_{i=-\infty}^{\infty} h_{i} y_{k-i} \\
& =h_{0} y_{k}+\sum_{i=-\infty}^{-1} h_{i} y_{k-i}+\sum_{i=1}^{\infty} h_{i} y_{k-i},
\end{aligned}
$$

where $h_{j}=h(j)$. 
The first term is the desired output quantity while the additional terms constitute undesired intersymbol interference. The second term in (3) depends on future data symbols and is caused by the nonvanishing samples of the overall impulse response for $i<0$ (precursors) while the third term is a function of previously detected data symbols and conceptually may be eliminated by decision feedback techniques (see [7]). This type of intersymbol interference is termed causal, because it is due solely to the postcursor samples of the impulse response. The prefilter which we are examining here can at most affect this causal intersymbol interference and for purposes of this study we shall assume that $h_{i}=0, i \leqslant-1$. [This can be achieved with a suitable linear digital filter at the receiver (see [5])]. Now if the values of $h_{i}$ are known at the transmitter, then a linear digital filter obeying the recursion

$$
y_{k}=x_{k}-\sum_{i=1}^{\infty} h_{i} y_{k-i}
$$

causes the complete elimination of postcursor intersymbol interference. (We have set $h_{0}=1$ without loss of generality). Clearly the difficulty with this linear scheme is that the output values $\left\{y_{k}\right\}_{0}{ }^{\infty}$ would be unbounded if the recursive filter with feedback weights $\left\{h_{i}\right\}$ were unstable and therefore the possibility of generating infinite power exists. In order to mitigate this situation, the nonlinear filter obeying the recursion in (1) is introduced. The object of course is to force the values of the output to be in approximately the same range as those of the input values.

To see how the nonlinear filter with bounded output power causes the elimination of intersymbol interference, consider an alternative way of expressing the recursion in (1)

$$
y_{k}=x_{k}-\sum_{i=1}^{N} \beta_{i} y_{k-i}+2 L n_{k}
$$

where $n_{k}$ is a suitable integer. Substituting (4) into (3) with $h_{0}=1$ yields

$$
\begin{aligned}
s_{k}= & x_{k}+\sum_{i=1}^{N} y_{k-i}\left(h_{i}-\beta_{i}\right) \\
& +\sum_{i=N+1}^{\infty} y_{k-i} h_{i}+2 L n_{k} .
\end{aligned}
$$

Now if $h_{i}=\beta_{i}, i=1, \cdots N$ and $h_{i}=0 i>N$ we see that

$$
s_{k}=x_{k}+2 L n_{k} \text {. }
$$

Since by hypothesis $x_{k} \in[-L, L]$, reducing $s_{k} \bmod 2 L$ yields $x_{k}$. This, then, is the key idea behind the precoding scheme. We also note here that reducing the range $(-L, L]$ of the function $f(u)$ would not be desirable for this would increase the vulnerability to noise for the extreme values $\pm(L-1)$ of $x_{k}$.

Summarizing briefly: Causal intersymbol interference can be completely eliminated by this nonlinear precoding tech-
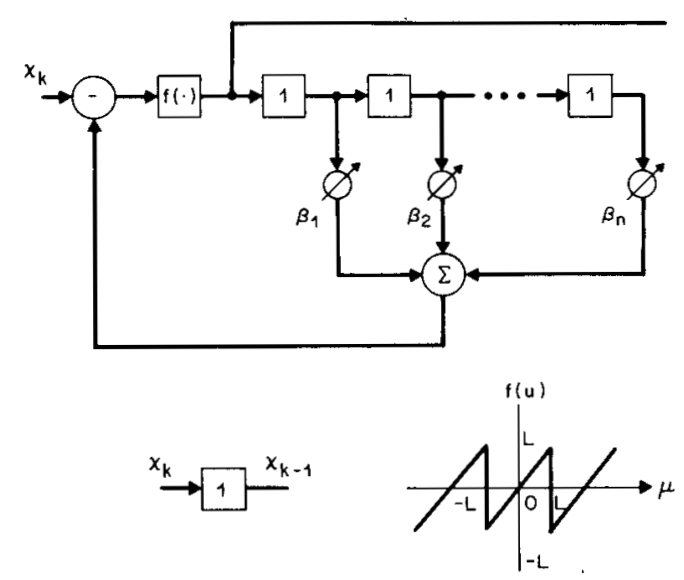

Fig. 1. Nonlinear baseband data precoding filter.

nique without incurring a noise penalty. It is known that the same can be achieved by decision feedback techniques at the receiver. But since this feedback must be done in the presence of noise, error propagation may result (for a discussion of decision feedback equalization and error propagation see Duttweiler et al. [8] ). We have seen that a linear filtering scheme at the transmitter could also eliminate postcursors while at the same time avoid error propagation, but the transmitted signal power may be unbounded. The chief advantage then of the nonlinear filter discussed here is that the transmitted symbols give rise to bounded power because they are restricted to the same range as the original data symbols. Our main concern here is to derive bounds on the transmitted signal power after precoding, and thus establish the penalty in transmitted signal power that must be paid for accomplishing the equalization at the transmitter. Since some data communication systems employ QAM, we extend this nonlinear precoding scheme to this modulation technique by generalizing to a two-input-twooutput coupled digital filter.

\section{THE CENTRAL RESULTS}

An interesting mathematical problem is to determine the probability distribution of $\left\{y_{k}\right\}_{1}^{\infty}$ obeying the nonlinear recursion (1). In general one would not expect a probability density to exist; the distribution could very well be singular. It is easy to demonstrate that $\left\{y_{k}\right\}_{1}^{\infty}$ is uniform when $\left\{x_{k}\right\}_{1}^{\infty}$ is uniform. Moreover, it can be shown that if the $x_{k}$ 's are independently and identically uniformly distributed, the $y_{k}$ 's are also independent and uniform. Halfin [9] has recently examined the first-order probability distribution when $\beta_{i}=0$, $i>1$. Our main concern however is to determine the average power in the transmitted signal ${ }^{2}$

$$
S(t)=\sum_{k=0}^{\infty} y_{k} g(t-k T)
$$

where $g(t)$ is a band-limited pulse satisfying $g(k T)=0, k \neq 0$

${ }^{2}$ Price has shown that the optimum transmitting filter shape is in fact flat across the Nyquist band. 
and $g(0)=1$. The average power is determined solely by $E y_{k}{ }^{2}$ (see Slepian [10]), on which we concentrate our attention. Recall that for the $L$-level $x_{k}$ variable

$$
E x_{k}^{2}=\frac{L^{2}-1}{3}
$$

We shall show that, independent of the (finite) values that the $\beta_{i}$ take,

$$
\frac{L^{2}-1}{3} \leqslant E y_{k}{ }^{2} \leqslant \frac{L^{2}-1}{3}+1 \quad k=1,2, \cdots .
$$

We begin by noting that (1) may be written

$$
y_{k}=f\left[\theta_{k}+x\right] \text {, }
$$

where $\theta_{k}$ is a symmetric random variable, independent of $x$, the latter being of the $L$-valued variety described above. Our approach is to find the distribution for $\theta_{k}$ that maximizes $E y_{k}{ }^{2}$ in (10). At this stage even the symmetry of $\theta_{k}$ will not be exploited; it will be shown later that including this requirement will not improve the bounds obtained. Clearly, if $F(\theta)$ is the distribution function of $\theta_{k}$; then

$$
E y_{k}^{2}=\frac{1}{L} \sum_{i=1}^{L} \int f^{2}\left[\theta+l_{i}\right] d F(\theta)
$$

where $l_{i}, i=1, \cdots, L$ indicates the $L$ values of $x$ [see (2)]. Since $\theta_{k}$ is bounded for any $k$, and $f^{2}$ is bounded, we can easily approximate the probability distribution of $\theta$ in (11) by a finite sum of delta functions

$$
\sum_{i} a_{i} \delta\left(\theta-\xi_{i}\right)
$$

so as to yield an arbitrarily accurate value for $E y_{k}{ }^{2}$. Note that the contribution of each delta function, generically called $a \delta(\theta-\xi)$, to $E y_{k}{ }^{2}$ is additive.

The key observation is that if one calculates the contribution of

$$
\begin{aligned}
& \quad a \delta(\theta-\xi) \quad-1 \leqslant \xi \leqslant 1 \\
& \text { to } E y_{k}^{2} \text {; then } \\
& \quad a \delta(\theta-\xi-2) \quad-1 \leqslant \xi \leqslant 1
\end{aligned}
$$

has the same contribution. To see this first consider, according to $(13)$, what $f(\xi+x)$ is. It is a random variable taking values

$$
-(L-1)+\xi,-(L-1)+\xi+2, \cdots,(L-1)+\xi
$$

with equal probability. Similarly, $f(\xi+2+x)$ for $\xi>0$ will be a random variable taking values

$$
\begin{aligned}
& -(L-1)+\xi+2,-(L-1)+\xi+4, \cdots, f[(L-1)+\xi+2] \\
& =-(L-1)+\xi
\end{aligned}
$$

with equal probability. Thus $f(\xi+x)$ and $f(\xi+2+x)$, and by the same argument $f(\xi \pm 2 j+x), j=$ integer, all have the same distribution, $|\xi|<1$. Hence, in finding $E y_{k}{ }^{2}$ we need only consider in (12) terms of the type (13) under the indicated restriction.

Now recalling again that $x$ takes the $L$ values $\pm(L-1)$, $\pm(L-3), \cdots$, consider a particular pair of these, $\pm K$. From (11), this pair yields a contribution to $E y_{k}^{2}$ which is proportional to

$$
(\xi+K)^{2}+(\xi-K)^{2}=2 \xi^{2}+2 K^{2} .
$$

It is evident that for $|\xi| \leqslant 1,(17)$ is minimized by $\xi=0$ and maximized by $\xi= \pm 1$, independently of $K$. Clearly then, the minimum value of $E y_{k}^{2}$ is obtained by taking $\xi=0, a=1$ in (13) to obtain

$$
E y_{k}^{2} \geqslant E x^{2}=\frac{L^{2}-1}{3},
$$

while taking $\xi=1, a=1$ in (13) yields

$$
E y_{k}{ }^{2} \leqslant \frac{L^{2}-1}{3}+1
$$

The result (19) is obtained most simply as follows. For $\xi=1$, we must evaluate

$$
E y_{k}^{2}=\frac{1}{L} \sum_{x}(1+x)^{2}
$$

where the sum over $x$ in (20) denotes the sum over the $L$ possible values that $x$ may have.

Since $\Sigma_{x} x=0,(20)$ yields

$$
E y_{k}^{2}=\frac{1}{L} \sum_{x}\left(1+x^{2}\right)=1+\frac{L^{2}-1}{3} \text {. }
$$

At this point we note that the symmetric distribution

$$
\frac{1}{2} \delta(\theta-1)+\frac{1}{2} \delta(\theta+1)
$$

for $\theta$ yields the same upper bound for the power and hence symmetrization of the density of $\theta$ will not improve the bound.

Equation (19) says that at most a $3 \mathrm{~dB}$ penalty is incurred for a binary system, $1.38 \mathrm{~dB}$ for a 3-level system, $0.8 \mathrm{~dB}$ for a 4-level one, etc. Since the largest uncertainty in the bound is for the binary case, let us consider this further. Consider the special binary case

$$
y_{k}=f\left[y_{k-1}+x_{k}\right], \quad y_{0}=0, \quad k=1,2, \cdots .
$$

We immediately have by direct iteration that the densities $p\left(y_{k}\right)$ are

$$
\begin{aligned}
& p\left(y_{1}\right)=\frac{1}{2} \delta\left(y_{1}-1\right)+\frac{1}{2} \delta\left(y_{1}+1\right) \\
& p\left(y_{2}\right)=\frac{1}{4} \delta\left(y_{2}-2\right)+\frac{1}{2} \delta\left(y_{2}\right)+\frac{1}{4} \delta\left(y_{2}+2\right) \\
& p\left(y_{3}\right)=\frac{1}{2} \delta\left(y_{3}-1\right)+\frac{1}{2} \delta\left(y_{3}+1\right) .
\end{aligned}
$$




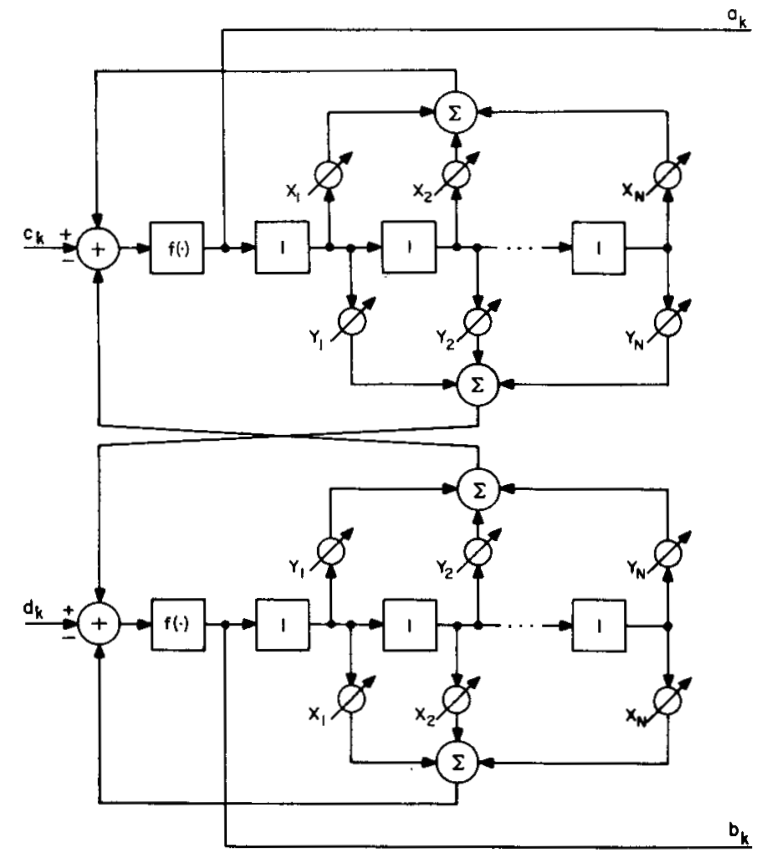

Fig. 2. Two-dimensional nonlinear precoding filter applicable in QAM.

Thus

$$
\begin{array}{ll}
E y_{k}^{2}=1, & k \text { odd } \\
E y_{k}^{2}=2, & k \text { even }
\end{array}
$$

which implies that the average power will be $(2+1) / 2$ times greater than without the nonlinear filter (or $1.76 \mathrm{~dB}$ greater). In summary, for the binary case there are situations where use of the nonlinear filter can incur a penalty of $1.76 \mathrm{~dB}$, but it cannot incur more than $3 \mathrm{~dB}$.

\section{GENERALIZATION TO QAM}

In QAM data transmission there are two independent streams of data symbols, $\left\{a_{k}\right\}_{0}{ }^{\infty}$ and $\left\{b_{k}\right\}_{0}{ }^{\infty}$, each transmitted on quadrature carriers. In addition to intersymbol interference, one also encounters cross-channel interference. Suppose $\left\{a_{k}\right\}_{0}^{\infty}$ is set over the in-phase channel and $\left\{b_{k}\right\}_{0}^{\infty}$ over the quadrature. After demodulation and sampling at time $k$, the pair of output values can be expressed as

$$
\begin{aligned}
& s_{k}(1)=a_{k}+\sum_{i=1}^{\infty} a_{k-i} x_{i}-\sum_{i=1}^{\infty} b_{k-i} y_{i} \\
& s_{k}(2)=b_{k}+\sum_{i=1}^{\infty} b_{k-i} x_{i}+\sum_{i=1}^{\infty} a_{k-i} y_{i}
\end{aligned}
$$

Ideally $s_{k}{ }^{(1)}$ should equal $a_{k}$ and $s_{k}{ }^{(2)}$ should equal $b_{k}$. In (26a) and (26b), $\left\{x_{i}\right\}^{\infty}$ and $\left\{y_{i}\right\}^{\infty}$ are the causal values of the overall in-phase and quadrature impulse responses, respectively.

The idea now is to design a two-input port and two-output port filter which encodes the pair of data sequences $\left\{c_{k}\right\}_{0}{ }^{\infty}$ and $\left\{d_{k}\right\}_{0}^{\infty}$ onto a pair $\left\{a_{k}\right\}_{0}^{\infty}$ and $\left\{b_{k}\right\}_{0}{ }^{\infty}$. Knowing what one is after, the invention comes relatively easy. A block diagram is shown in Fig. 2. As seen the output symbols satisfy the recursions

$$
\begin{aligned}
& a_{k}=f\left[c_{k}-\sum_{i=1}^{N} a_{k-i} x_{i}+\sum_{i=1}^{N} b_{k-i} y_{i}\right] \\
& b_{k}=f\left[d_{k}-\sum_{i=1}^{N} b_{k-i} x_{i}-\sum_{i=1}^{N} a_{k-i} y_{i}\right]
\end{aligned}
$$

where $f(\cdot)$ is defined as before.

It can be verified that when $(27 \mathrm{a})$ and $(27 \mathrm{~b})$ are substituted into (26a) and (26b), respectively, and the results reduced $\bmod 2 L, s_{k}^{(1)}=a_{k}$ and $s_{k}(2)=b_{k}$. As in the baseband case the transmitted power is determined by $E a_{k}{ }^{2}$ and $E b_{k}{ }^{2}$ since the signals are sent on orthogonal carriers. Because there is no cross coupling between the channels, we need make no additional arguments about the bounds on the transmitted power and they are the same here as in the baseband situation.

\section{REFERENCES}

[1] H. Miyakawa and H. Harashima, "Capacity of channels with matched transmission technique, for peak transmitting power limitation," in Nat. Conv. Rec. Inst. Elec. Commun. Eng. Japan, Aug. 1969, no. 1268, p. 1269.

[2] M. Tomlinson, "New automatic equalizer employing modulo arithmetic," Electron. Lett., vol. 7, pp. 138-139, Mar. 1971.

[3] E. R. Kretzmer, "Binary data communication by partial response transmission," in Conf. Rec., First IEEE Annu. Commun. Conv., June 1965, p. 451.

[4] A. M. Gerrish and R. D. Hanson, "Multilevel partial response signaling," in 1967 IEEE Int. Conf. Commun. Rec., Minneapolis, MN, p. 186.

[5] R. Price, "Nonlinearly feedback-equalized PAM vs. capacity for noisy linear channels," in Conf. Rec., IEEE Int. Conf. Commun., Philadelphia, PA, June 19-21, 1972.

[6] D. G. Messerschmitt, "Generalized partial response for equalized channels with rational spectra," IEEE Trans. Commun., vol. COM-23, pp. 1251-1258, Nov. 1975.

[7] J..Salz, "Optimum mean-square decision feedback equalization," Bell Syst. Tech. J., vol. 52, no. 8, Oct. 1973.

[8] D. L. Duttweiler, J. E. Mazo, and D. G. Messerschmitt, "On the error probability of decision-feedback equalization in the presence of error propagation," IEEE Trans. Inform. Theory, vol. IT-20, pp. 490-497, July 1974.

[9] S. Halfin, "Explicit construction of invariant measures for a class of continuous state Markov processes," Ann. Probability, to be published.

[10] D. Slepian, "On maxentropic discrete stationary processes," Bell Syst. Tech. J., vol. 51, no. 3, Mar. 1972.

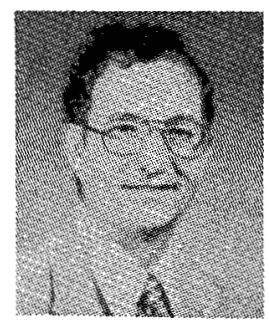

James E. Mazo (M'65) received the B.S. degree in physics from the Massachusetts Institute of Technology, Cambridge, in 1958, and the M.S. and Ph.D. degrees in physics from Syracuse University, Syracuse, NY, in 1960 and 1963 , respectively.

Upon completion of his studies, he was employed as a Research Associate in the Department of Physics, University of Indiana, Bloomington, where he did theoretical studies in scattering theory. In 1964 he joined Bell Laboratories, Holmdel, NJ, engaging in various theoretical problems concerned with data transmission. Since 1972 he is with the Mathematical Research Member of Bell Laboratories, Murray Hill, NJ.

Dr. Mazo is a member of the American Physical Society. 


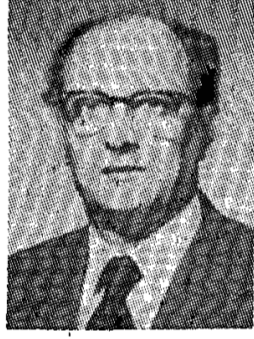

Jack Salz was born in Mukach, Czechoslovakia, on March 14, 1930. He received the B.S., M.S., and $\mathrm{Ph} . \mathrm{D}$. degrees in electrical engineering from the University of Florida, Gainesville, in 1955 , 1956 , and 1961, respectively.

From 1956 to 1958 he was a Member of the Technical Staff at Bell Laboratories, Whippany, NJ. During this time he participated in the Communications Development Training Program and worked on the electronic switching system. From 1958 to 1962 he was employed by the Martin Company, Orlando, FL. From 1958 to 1959 he did research on various theoretical communication problems; from 1959 to 1960 he was a Consultant while completing his Ph.D. degree; and from 1960 to 1962 he was Head of a group concerned with fundamental theoretical studies of communication and radar systems. Since 1962 he has been with Bell Laboratories, Holmdel, NJ, except during 1967-1968 when he was Professor of Electrical Engineering, University of Florida. At present he is a Supervisor in the Data Theory Department, where he is concerned with various technical problems in the area of data communication.

Dr. Salz is a member of Sigma Xi.

\section{Concise Papers}

\section{A Simulation Model of the TIDAS Computer Network}

\section{TORSTEN CEGRELL}

Abstract-To study the performance of a full-duplex messageswitched computer network, called TIDAS-T, during various conditions a simulation model has been built in order to simulate the procedure. The model describes the communication and the line procedure in the TIDAS network very closely. The only simplification made is that the processing time in the node computers is disregarded as being insignificant in comparison with the transmission and queueing times. The model, programmed in SIMSCRIPT 1.5 , is very flexible because all important parameters are given by input data.

The outputs from a simulation differ from run to run due to the object defined by input data parameters. The statistical results are mostly presented as complete statistical distributions.

It has turned out that the model has been a splendid tool in order to study and view the consequences of different design approaches.

Some of the problems in building such a complex model are elucidated and șome simulation runs are given as illustrations.

As the model is very flexible it may be used to study computer networks other than TIDAS.

\section{INTRODUCTION}

The growth of the power system in Sweden, with many new power generating stations and a more complex power transmission network, has caused the Swedish State Power Board to build an information system including automatic data acquisition and transmission of information as well as on-line control, security monitoring, and planning, to keep the high security and quality of electric power supply.

This system, called TIDAS [1], [2], and [7], which is now under construction and covers all of Sweden, consists of two subsystems, a data processing system, TIDAS-D, and a data transmission system, TIDAS-T, see Fig. 1 .

The interest in this concise paper will only be given to the T-system, which handles all the communications and supplies the data bases in the $D$-system or in the regional control centers with suitable data and transfers, e.g., set points and control orders for breakers out in the power system.

The T-system consists of a number of so-called collecting points. A collecting point is built up around one or two minicomputers. The different collection points are connected

Paper approved by the Associate Editor for Computer Communication of the IEEE Communications Society for publication after presentation at the International Symposium SIMULATION '75, Zurich, Switzerland, June 23-26, 1975. Manuscript received June 9, 1975; revised October 10, 1975.

The author is with the Electronics Division, ASEA, Västerạ̊s, Sweden.

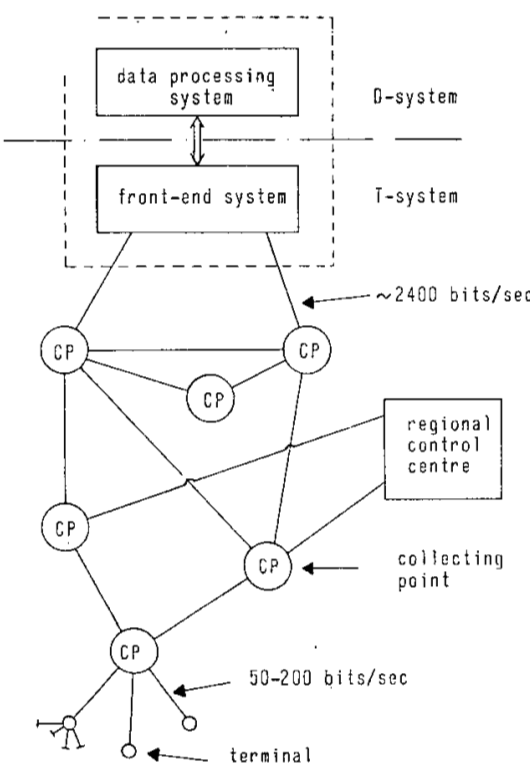

Fig. 1. Principle scheme of the TIDAS information system.

to each other over a complex distributed medium speed full-duplex transmission network.

To a collecting point a number of substations or terminals are also connected. The terminals are often placed in power generating stations or in power transformer stations within a limited area around a collecting point. The communication to the collecting point operates in half-duplex mode on fullduplex low speed transmission lines.

The communication lines consist chiefly of carrier circuits on power lines and to a less extent of radio links and cables.

To give a feeling of the size of the actual T-system some rough figures are given in the following. There will be about 20 nodes, the main part working as collecting points and some working as front ends, and about 200 terminals. The amount of information is difficult to illustrate in terms of numbers. However, there will be thousands of indications from, e.g., breakers, analog measured values (megawatts, megavolt amperes, and kilovolts), and digital measured values (water level, megawatt hours) to take care of.

The traffic exchange and the line procedure will now be briefly described and in Section I-B the modeling of the computer network is introduced.

\section{A. Message Switching}

Collecting points and front-end systems are all nodes in the data transmission network. In this network, traffic exchange is 